\title{
Learning in human neural networks on microelectrode arrays
}

\author{
R. Pizzi ${ }^{a}, *$ G. Cino ${ }^{\text {a }}$, F. Gelain ${ }^{\text {b, } 1}$, D. Rossetti ${ }^{a}$, A. Vescovi ${ }^{b}$ \\ ${ }^{a}$ Department of Information Technologies, University of Milan, via Bramante 65, 26013 Crema (CR), Italy \\ b Stem Cells Research Institute, DIBIT S. Raffaele, via Olgettina 58, 20132 Milano, Italy \\ Received 19 July 2005; received in revised form 10 February 2006; accepted 3 March 2006
}

\begin{abstract}
This paper describes experiments involving the growth of human neural networks of stem cells on a MEA (microelectrode array) support.

The microelectrode arrays (MEAs) are constituted by a glass support in which a set of tungsten electrodes are inserted.

The artificial neural network (ANN) paradigm was used by stimulating the neurons in parallel with digital patterns distributed on eight channels, then by analyzing a parallel multichannel output. In particular, the microelectrodes were connected following two different architectures, one inspired by the Kohonen's SOM, the other by the Hopfield network.

The output signals have been analyzed in order to evaluate the possibility of organized reactions by the natural neurons.

The results show that the network of human neurons reacts selectively to the subministered digital signals, i.e., it produces similar output signals referred to identical or similar patterns, and clearly differentiates the outputs coming from different stimulations.

Analyses performed with a special artificial neural network called ITSOM show the possibility to codify the neural responses to different patterns, thus to interpret the signals coming from the network of biological neurons, assigning a code to each output. It is straightforward to verify that identical codes are generated by the neural reactions to similar patterns.

Further experiments are to be designed that improve the hybrid neural networks' capabilities and to test the possibility of utilizing the organized answers of the neurons in several ways.
\end{abstract}

(C) 2006 Elsevier Ireland Ltd. All rights reserved.

Keywords: Neural networks; Stem cells; Microelectrode arrays; Learning; Self-organization

\section{Introduction}

During the past decade several laboratories have conducted experiments on direct interfacing between electronics and biological neurons in order to support neurophysiological research and also to pioneer future hybrid human-electronic devices, bioelectronic prostheses, bionic robotics, and biological computation.

\footnotetext{
* Corresponding author. Tel.: +3902 50330072; fax: +390250330010 .

E-mail addresses: pizzi@dti.unimi.it (R. Pizzi), gelain.fabrizio@hsr.it (F. Gelain).

1 Tel.: +3902 26434952 .
}

As microelectrodes implanted into brain give rise to rejection and infections, a direct adhesion between electronics and neural tissue has been experimented, achieving important results (Egert et al., 1988; Akin et al., 1994; Wilson et al., 1994; Breckenridge et al., 1995; Bove et al., 1996; Weis et al., 1996; Borkholder et al., 1997; Canepari et al., 1997; Jenkner and Fromherz, 1997; Schatzthauer and Fromherz, 1998; Maher et al., 1999; Jimbo and Robinson, 2000; Reger et al., 2000; School et al., 2000; Braun and Fromherz, 2001; Jenkner et al., 2001; Potter, 2001; Zeck and Fromherz, 2001; Bels and Fromherz, 2002; Bonifazi and Fromherz, 2002).

During the early 1990s a direct interface between nervous cells and silicon was established. In particular leech 
neurons were used because of their big size (Fromherz et al., 1991). The Fromherz's group (Max Planck Institute of Biochemistry) first pioneered the silicon/neuron interface and keeps developing sophisticated techniques to optimise this kind of junction (Fromherz et al., 1993; Fromherz and Schaden, 1994; Fromherz, 2002).

Many other experiments have been conducted, with different aims: in 1999, William Ditto and collaborators at the Georgia Tech tried to obtain simple computations from a hybrid leech-electronics creature. As all the physiological signals can be seen as chaotic time series, they used chaos theory to interpret the neural output (Schiff et al., 1994; Lindner and Ditto, 1996; Garcia et al., 2003).

In 2000, a team of the Northwestern University of Chicago, University of Illinois and University of Genoa (Reger et al., 2000) developed a hybrid creature consisting of lamprey neurons connected to a robot. When presented with light stimuli, the creature behaves in different ways: follows light, avoids it, and moves in circle.

In 2002, Steve Potter (Georgia Tech) created a hybrid creature made by few thousand living neurons from a rat cortex placed on a special glass Petri dish instrumented with an array of 60 micro-electrodes, that was also able to learn from environment (De Marse et al., 2002).

In 2003, the Duke University's group (Carmena et al., 2003) succeeded in connecting 320 micro-electrodes to monkey cells in the brain, allowing to translate the electrical signals directly into computer instructions, that were able to move a robotic arm. This will be a way to allow disabled people to move paralyzed limbs or electronic prostheses.

Despite these astonishing results, neurophysiological research is far from a detailed understanding of the learning mechanism of the brain and fails to interpret the cognitive meaning of the signals coming from the neurons. A deeper and innovative analysis of the signals coming from a direct connection between electronics and neural tissue could disclose new prospects in this field. On the other hand, a correct interpretation of the signals could support a faster development of the abovedescribed bionic techniques.

With this purpose, our group, formed by researchers of the Department of Information Technologies of the University of Milan and of the Stem Cells Research Institute of the DIBIT-S. Raffaele Milan, is experimenting with the growth of human neural networks of stem cells on a MEA (microelectrode array) support.

In order to examine neural learning and memorizing activities, we developed architectures based on artificial neural network models on networks of human neural stem cells adhering to microelectrode arrays (MEAs).
The MEAs are connected to a $\mathrm{PC}$ via an acquisition device that allows stimulating the neurons with suitable inputs and acquiring the neuron signals in order to evaluate their reactions.

In this paper we describe the techniques we used to perform this task.

By stimulating cells with digital bitmap patterns, we recorded their electrical reactions and examined the output signals with advanced techniques. This allowed us to evaluate the self-organization of the biological neural networks during and after the learning, and to discriminate their reactions to different patterns.

\section{Materials and methods}

\subsection{The stem cells}

Our neurons have been cultured starting from human neural stem cells extracted by a human embryo. Stem cells are multipotential undifferentiated cells whose main features are the ability of self-renewal and of differentiation into several types of adult cells (Vescovi et al., 1999; Gritti et al., 2001).

We chose to adopt human stem cells because their wellknown capability to integrate with the host tissue in transplantation procedures. This could allow a future direct implantation of a bionic chip into human neural tissue without rejection.

Moreover, stem cells have the advantage to develop a cellular line, thus a virtually infinite number of standardized cells, whereas the use of other types of cells does not ensure the same behavior at every experiment.

Furthermore, stem cells allow developing more phenotypes (not only neurons but also astrocytes and oligodendrocytes) that ensure the correct contribution of trophic substances and cellular junctions for a better and more physiological functionality of neurons in culture.

Their multipower and extreme plasticity leads us to believe that interesting results could be drawn by their organized stimulation. Stem cells grow into adult neurons in about one month, developing all the essential properties of functional CNS neurons (Gritti et al., 2000; Song et al., 2002).

On the other hand, the culture of human neural stem cells is very delicate and different both from the culture of animal stem cells and of neural brain slices, requesting an extremely specialized knowledge.

Therefore our culture method on MEA is quite different from those reported in the previous paragraph. Nevertheless, the culture method adopted in our experiments has been well established in time by Prof. Angelo Vescovi's team (Gritti et al., 2001).

Cells are plated at a density of 3500 cells $/ \mathrm{cm}^{2}$ in suspension in a chemically defined, serum-free medium containing $20 \mathrm{ng} / \mathrm{mL}$ of human recombinant epidermal growth factor (EGF) and $10 \mathrm{ng} / \mathrm{mL}$ of fibroblast growth factor (FGF-2). After 3-5 days the cultures are harvested and the cells are mechanically dissociated and replated under the same conditions. Our 
experiments have been performed 4 weeks after seeding our neural stem cells onto MEA surfaces previously coated with adhesive substrates, like mouse Laminin ( $2 \mathrm{ng} / \mathrm{ml})$ and human fibronectin $(2 \mathrm{ng} / \mathrm{ml})$.

Each experiment requires the culture of several MEAs, and the correct growth and adherence to the electrodes are controlled daily. Measures are performed only on the MEAs that present optimal adhesion and correct growth.

In order to ensure that the collected signals are actually due to electrophysiological functionalities of neurons, every experiment is equipped by measures from control basins with cultured fibroblasts to compare behavior discrepancies. Moreover, at the end of the experiments the cultures are injected with Tetrodotoxin (TTX), a neurotoxin able to abolish action potentials. Then TTX is rinsed away ( 3 rinses $\left.\times 5^{\prime}\right)$ and after 5 min the same measures are repeated. All the control procedures confirmed the presence of neural electrical activity.

\subsection{The hardware}

The problem of the junction between neuron and electrode is crucial: materials must be biocompatible with the culture environment, and neurons must adhere firmly to the electrodes in order to promote growth and to ensure good stimulation and recording.

Our supports are constituted by glass dishes with 96 tungsten microelectrodes (Fig. 1).

Each electrode is connected, by means of $20 \mu \mathrm{m}$ wide electrodes, to a pad that is suitable for the external connection.

During the last decade several commercial MEAs have been proposed. Our MEA set is a prototype with electrodes of different width that is suitable to experiment the adhesion of the neural stem cells, whose special culture method causes adhesion properties different from those of the known adult neural cells.

The distance between electrodes varies between 100 and $200 \mu \mathrm{m}$, whereas the diameter of each electrode is around $20 \mu \mathrm{m}$. During the experiments only distant electrodes have been used, thus the actual distance between electrodes ranged between 300 and $500 \mu \mathrm{m}$.
On the MEA four plastic rings have been past using a nontoxic silicone, in such a way as to realize four basins and arrange more experiments simultaneously.

From the 96 electrodes some have been chosen as neural input/output, others as ground.

The electrodes have been connected to realize special layouts, as described in the next paragraph.

The block diagram of the hardware is represented in Fig. 2.

The whole circuit is composed by:

- Electrodes shared among four basins in which cells are cultured.

- A shielded cable used to send and receive electrical signals between electrodes and stimulation circuit.

- Two shielded cables used to send analog signals to the acquisition card and to transfer the digital signals coming from the I/O ports of the acquisition card to the stimulation circuit.

- National Instruments NI6052E DAQ acquisition card, $333 \mathrm{kHz}$, optically shielded, 16 analog inputs, two analog outputs, and eight digital I/O lines.

- An electronic stimulation circuit whose task is to convert digital eight-bit signals (patterns), generated by the software resident on the PC, from electrical signals generated by the acquisition card with a logical level $0-5 \mathrm{~V}$, into electrical pulses with voltage and current suitable to stimulate neurons.

The stimulation occurs with a $35 \mathrm{mV}$ positive voltage. In order to depolarise the cultured neurons, before every bit, a negative $-35 \mathrm{mV}$ pulse is emitted. The pulse length is $10 \%$ of the whole bit duration, thus the whole pulse is composed by $10 \%$ negative voltage, $90 \%$ positive voltage.

In order to experiment the cell reactivity to electrical stimulation, we stimulated the cells with frequency bursts varying from 40 to $800 \mathrm{~Hz}$; therefore the whole pulse duration was between 1.25 and $25 \mathrm{~ms}$ (see Fig. 7).

The computer's task is to generate the patterns and store on the hard disk the data coming from the cells.

To avoid interference, the stimulation circuit disconnects the voltage generator when the acquisition card gets ready to receive the signals coming from the culture basins.
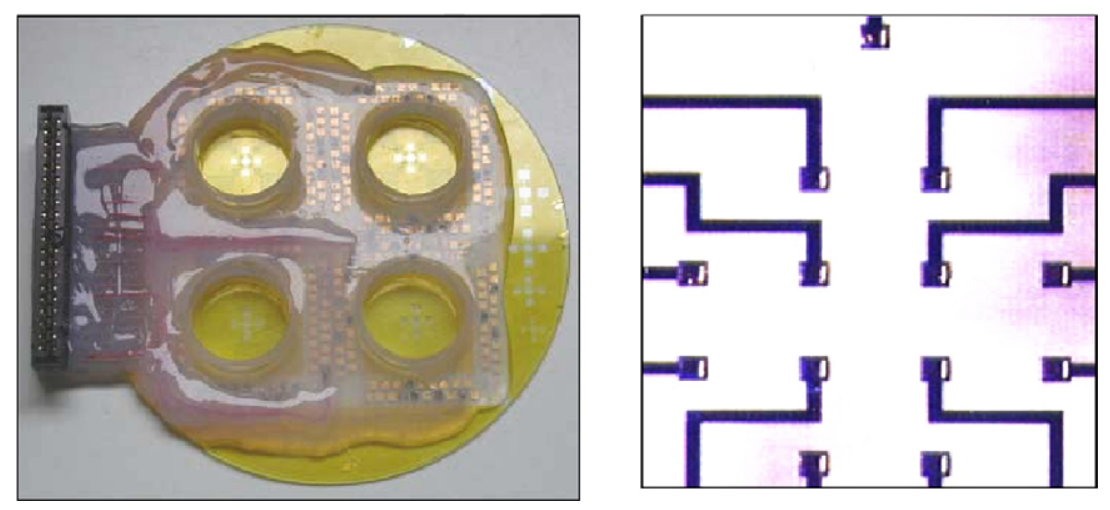

Fig. 1. The MEA support. On the left, the whole support with the four basins, on the right an enlargement of the electrodes. 


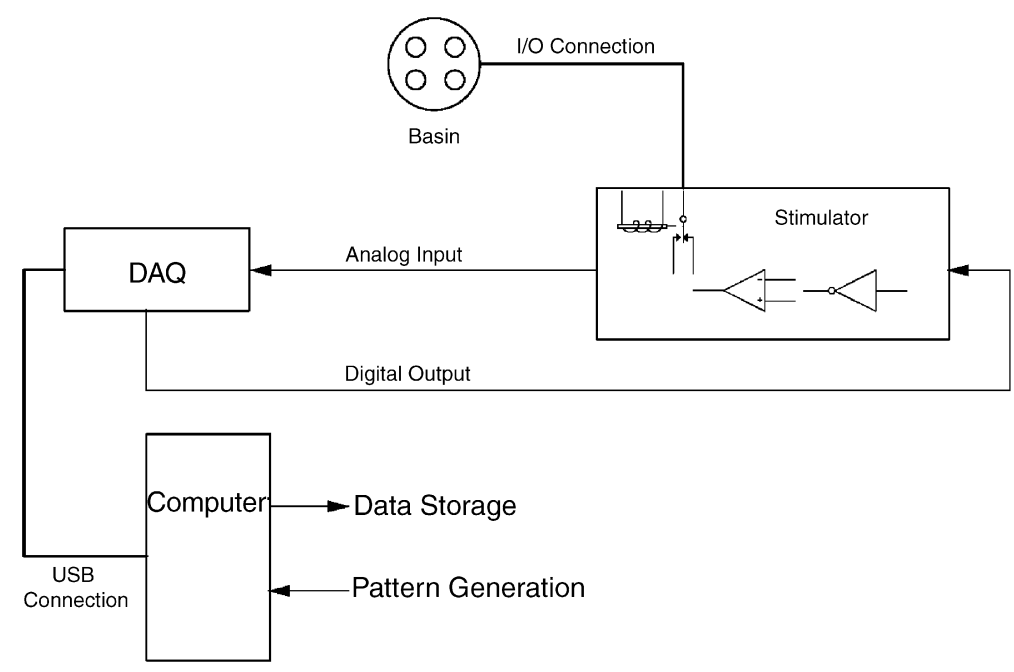

Fig. 2. Block diagram of the hardware: acquisition and stimulation systems. The stimulator injects digital patterns to the neurons, then receives their electrical reaction and passes the signals to the acquisition card (DAQ).

The electronic circuit is included in a plastic box whose walls have been treated with special varnishes that efficiently shield possible EMI (electrical magnetic interference) noise. In addition, a $50 \mathrm{~Hz}$ Notch filter has been inserted (European electrical supply frequency).

All the cables used for the connection between culture basins, stimulation circuit, and acquisition card have been shielded carefully. We also minimized the power supply ripple using a capacitor with low equivalent-series-resistance (ESR), in order to avoid a possible ripple in the generated signals (Fig. 3).

\subsection{The artificial architecture}

On the MEAs we implemented two connection schemata resembling two artificial neural networks (ANN) architectures (Mc Culloch and Pitt, 1943; Rosenblatt, 1958; Rumelhart and Mcclelland, 1986; Haken, 1991; Haykin, 1994): a Kohonen

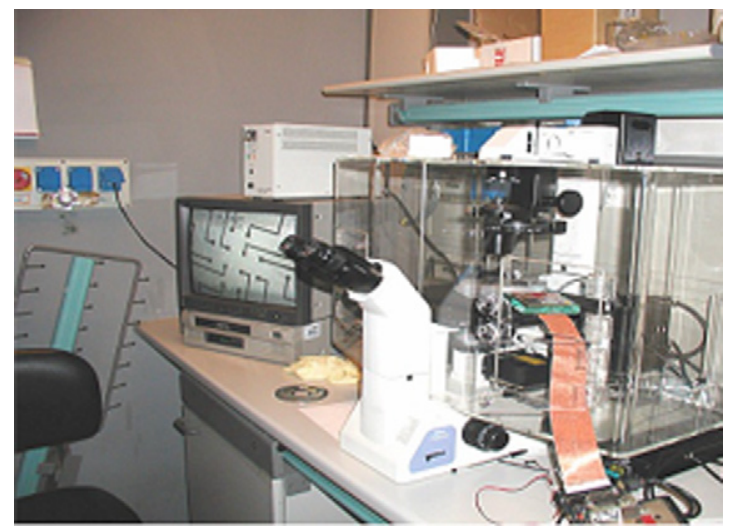

Fig. 3. Experimental setup: microscope with videorecorder, cells in incubator and cables to the hardware-software system. “self-organizing map" (Kohonen, 1990) and a Hopfield network (Hopfield, 1984).

In the case of the Kohonen network we arranged eight input channels picked from eight electrodes, on which living cells were attached. These cells were connected each other and to other cells to form a kind of "competitive layer". From other cells of this network, adhering to other electrodes, three output channels were picked.

In the case of the Hopfield network, we arranged eight input channels picked from eight electrodes, on which living cells were attached. The cells were connected each other as in the case of the Hopfield network. As in the Hopfield network's model, the output channels coincide with the input channels, thus after a short relaxation time, the output signals were collected from the same electrodes.

The networks' configuration was determined on the basis of a preliminary study, where two software artificial neural networks (Kohonen and Hopfield) have been implemented, in order to determine the minimum number of nodes able to exactly recognize simple bitmaps like those represented in Figs. 8 and 9.

The software simulation has highlighted that this minimum configuration is represented by eight input neurons and three output neurons for the Kohonen networks, and eight input/output neurons for the Hopfield network.

Adopting this "artificial" model we could apply a digital parallel input to the biological network, and our challenge was to verify if, as in the ANN paradigm, the answer given by the network of biological neurons showed an organized and selective reply as in the case of the software simulation.

\subsubsection{The self organizing map}

Physiological researches of mammalian sensory cortical areas (Mountcastle, 1957; Hubel and Wiesel, 1962, 1968, 1974) have established that neurons respond selectively to combinations of specific stimulus features. These preferences 


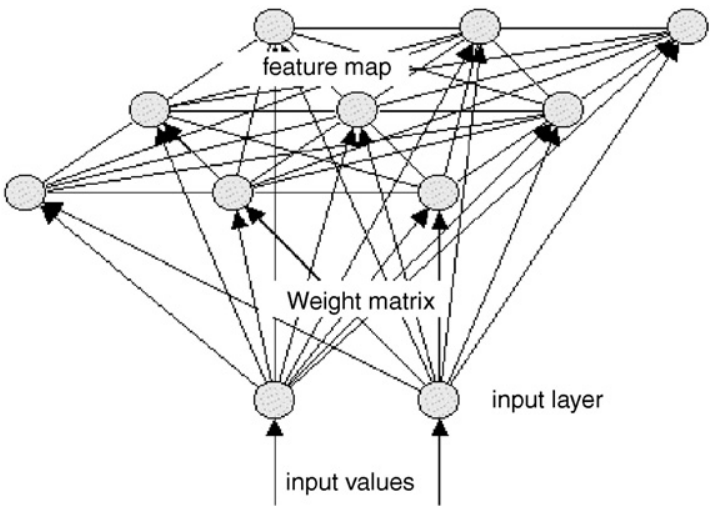

Fig. 4. Kohonen network (SOM). The output layer, after a "competition" with the neurons of the input layer, forms a map topologically similar to the input pattern.

usually, although not always, change smoothly with tangential position in the cortex.

Thus the cortical areas can be seen as a massively interconnected set of elementary processing elements (the so-called cortical "columns") that constitute what was called a "computational map" (Knudsen et al., 1987). The layout of many cortical areas shows a kind of topologic organisation that was often associated with a dimensionality reduction of the representational space (Durbin and Mitchinson, 1990).

The Kohonen Network (Self Organizing Map, SOM) was developed in the 1980s by T. Kohonen on the basis of the above-cited neurophysiological studies (Saarinen and Kohonen, 1985).

The SOM is a non-supervised network, i.e., it works without need of presentation of known examples.

The network structure consists of an input layer and a socalled competitive layer with $N$ neurons. Each of them receives $n$ signals $x_{1}, \ldots, x_{n}$ coming from the $n$ elements of the input layer, following connections with $w_{i j}$ weight (Fig. 4).

The intensity $I$ of the element $i$ is calculated by:

$I_{i}=D\left(w_{i}, x\right)$

where $D\left(w_{i}, x\right)$ is a distance function, for example the Euclidean one, between the input and each neuron of the competitive layer.

The learning phase (winner-take-all law, WTA) consists of a competition to evaluate which element has the minimum input intensity, i.e., which $w_{i}$ is the nearest to $x$.

The weights are modified following the law:

$w_{\text {inew }}=w_{\text {iold }}+\eta\left(x-w_{\text {iold }}\right) z_{i}$

where $0<\eta<1$ is the learning rate.

In this way the network moves more and more towards the nearest stimuli, ideally up to overlap them: the SOM performs a mapping from a multidimensional space to a space with fewer dimensions, preserving the starting topology: in other words, it classified a pattern as the nearest among a set of reference elements.

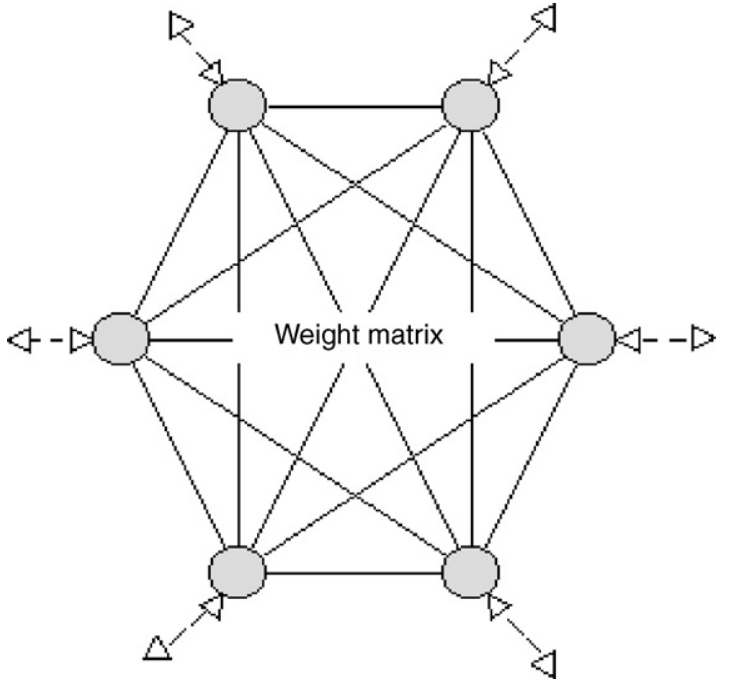

Fig. 5. Hopfield network. The neurons are all interconnected and they act both as input and as output nodes.

\subsubsection{The Hopfield network}

From 1982 to 1985 the physicist J.J. Hopfield presented a neural network model with an interesting architecture from a neurodynamical point of view.

The Hopfield network allows to memorize vectors and to recall them later.

The network consists of $n$ fully connected neurons, as shown in Fig. 5.

Input is applied simultaneously to all neurons; they interact with each other and the process continues until a stable state is reached, that represents the network output.

The units in the Hopfield networks are binary threshold units, i.e., the units only take on two different values for their states and the value is determined by whether or not the units' input exceeds their threshold. Hopfield networks can either have units that take on values of 1 or -1 , or units that take on values of 1 or 0 , or also real values between 1 and -1 . So the unit $i$ 's activation values are

$$
s\left(x_{i}\right)= \begin{cases}x_{i} & \text { if } \sum_{j \neq k} w_{i j} x_{i}=T_{i} \\ +1 & \text { if } \sum_{j \neq k} w_{i j} x_{i}>T_{i} \\ -1 & \text { if } \sum_{j \neq k} w_{i j} x_{i}<T_{i}\end{cases}
$$

where $w_{i j}$ is the connection weight from unit $j$ to unit $i$; $s\left(x_{i}\right)$ the state of the unit $i ; T_{i} \geq 0$ is the threshold of unit $i$.

The connections in a Hopfield network have two restrictions on them:

$w_{i i}=0, \quad \forall i$ (no unit has a connection with itself),

$w_{i j}=w_{j i}, \quad \forall i, j$ (all connections are symmetric).

We can see an input pattern as a point in the state space that, while the network is running, moves towards the minima 
representing the steady states of the network, in the lowest points of its attraction basins (Tank and Hopfield, 1989). When the network stops, the weights values are the network output.

If we associate to the network an energy function $E$ :

$$
E(x)=-\frac{1}{2} \sum_{i} \sum_{j \neq i} w_{i j} x_{i} x_{j}
$$

this value decreases monotonically in time.

In fact, being in this case $\Delta E / \Delta x_{i}=-\sum_{j} w_{i j} x_{j}$

$$
\begin{array}{ll}
\text { if } \Delta x_{i}>0 \quad \sum_{j} w_{i j} x_{j}>0 \\
\text { if } \Delta x_{i}<0 \quad \sum_{j} w_{i j} x_{j}<0
\end{array}
$$

i.e., always $\Delta E \leq 0$.

Thus after a number of iterations the network stabilizes into a minimum energy state.

Each minimum corresponds to a stored pattern.

The $m$ memorized forms $s_{1}, \ldots, s_{m}$ correspond to the local minima of the $E(s)$ function.

Therefore if we present to the network a vector $s^{\prime}$ slightly different from the stored patterns $s$, the network dynamics will relax on the local minimum nearest to $s^{\prime}$, i.e. $s$.

\subsection{The hybrid networks}

The artificial models have been implemented on the MEAs, culturing the stem cells on the connection sites.

Among the available electrodes we chose to connect to the acquisition card those on which the microscope showed 1-3 well-grown and living cells on the electrode or in close proximity of it (see Fig. 6):

(1) The Kohonen network is implemented by eight electrodes that constitute the input layer, and three electrodes that con-

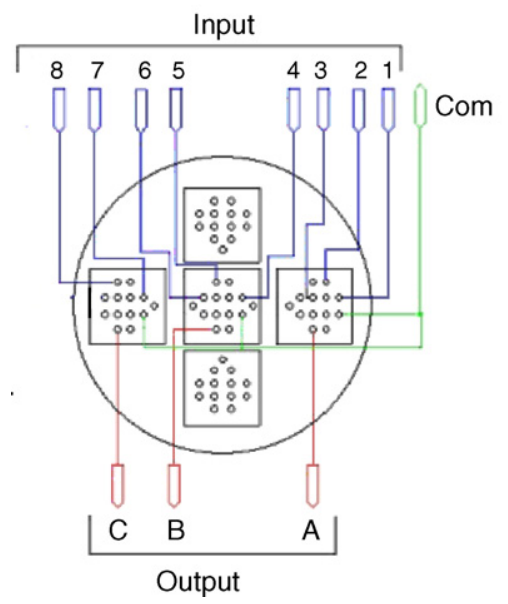

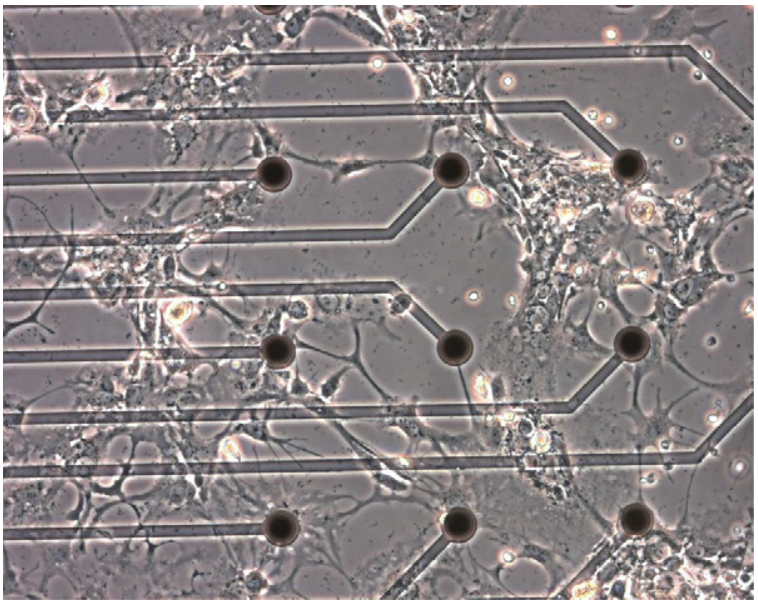

Fig. 6. Living stem cells on our MEA.

stitute the competitive layer. The input and the competitive electrodes are connected following the classical Kohonen architecture. The output signals are collected directly from the competitive layer.

(2) The Hopfield network is set up by eight electrodes acting both as input and as output (Fig. 7), following the classical Hopfield architecture.

These architectures have been simulated by a software artificial neural network and they have shown to constitute the minimum configuration suitable to correctly recognize two different input bitmaps (patterns), namely a " 0 " and a " 1 " constituted by nine-element bitmaps. The central value of the bitmap was always considered null for sake of electronic simplicity, obtaining eight-element bitmaps.

The patterns have been delivered to the hybrid networks as a train of electrical pulses in such a way as to represent every black square of the bitmap (see Fig. 8) as a $35 \mathrm{mV}$ pulse

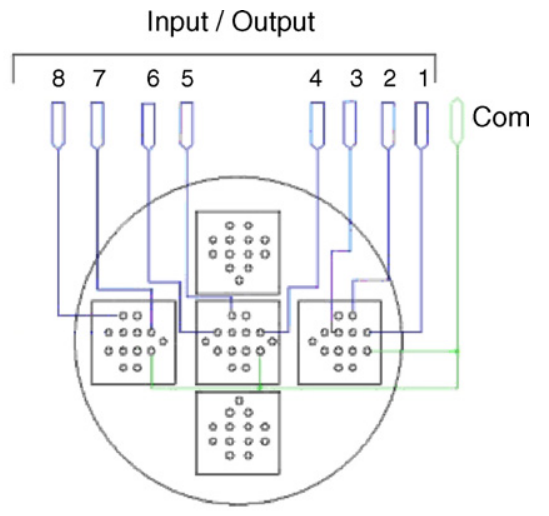

Fig. 7. Connections for the Kohonen network (left) and the Hopfield network (right) on MEA. In the left basin eight input electrodes and three output electrodes were connected. In the right basin eight input/output electrodes were connected. 


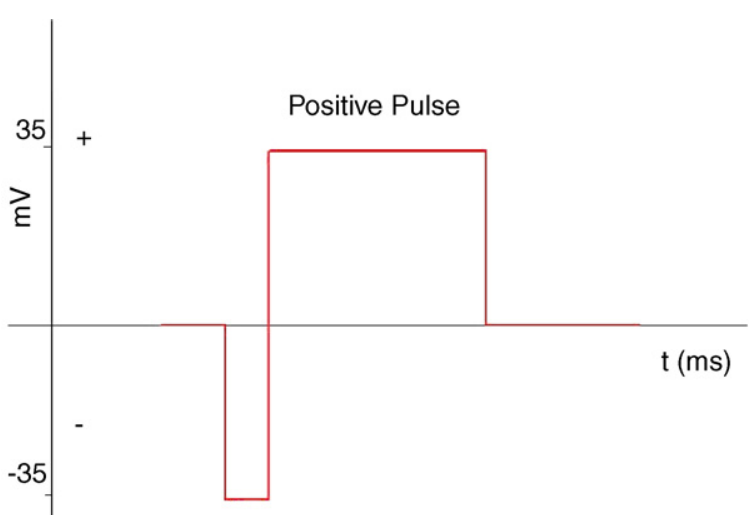

Negative Pulse

Fig. 8. Waveform of the stimulation pulse. The pulse duration was varied from 1.25 to $25 \mathrm{~ms}$.

(similar to the natural action potential) and every white square as a $0 \mathrm{mV}$ pulse.

Artificial neural networks and the human brain are able to recognize not only sharp images, but also images affected by noise: for this purpose we delivered to the hybrid networks also noise-affected patterns (Fig. 9a and b) in order to verify their ability to recognize them correctly.

In the last decade several authors supported the hypothesis that electrical correlated activity at around $40 \mathrm{~Hz}$ (gamma oscillations) frequency flows following sensory stimuli along the cortical neurons: "induced rhythms" at 50-60 Hz were first described in olfactory bulb (Adrian, 1950), then in olfactory (Eeckman and Freeman, 1990), visual (Eckhorn et al., 1998; Eckhorn et al., 1990; Engel et al., 1991a,b; Freeman and van Dijk, 1987; Gray et al., 1989; Singer and Gray, 1995), auditory (Pantev et al., 1991; Madler et al., 1991), somatosensory (Bouyer et al., 1987) and motor cortex (Murthy and Fetz, 1992; Sanes and Donoghue, 1993; Pfurtscheller et al., 1994). Gamma oscillations also occur in the hippocampus (Stumpf, 1965; Bragin et al., 1995).

In humans, brief " $40 \mathrm{~Hz}$ transient responses" (Tiitinen et al., 1993) increase when the subject pays attention and disappear with loss of consciousness during anaesthesia (Kulli and Koch, 1991). Repetitive auditory stimulation at $40 \mathrm{~Hz}$ generates a large "40 Hz steady state response" (Galambos et al., 1981). Other studies suggest that gamma rhythms may be organized to sweep across the whole brain, perhaps providing temporal binding into a single cognitive experience (Llinas and Ribary, 1993).

Our experience (Pizzi et al., 2002) has supported the hypothesis, maintained by the work of several authors (Freeman, 1987, 1994; Varela, 1995; Menon and Freeman, 1996; Chrobak and Buzsaki, 1998; Mitner et al., 1999; Dickson et al., 2000) that electrical correlated activity at $\sim 40 \mathrm{~Hz}$ has the possible aim (or effect) to create a functional binding of perceptions, thus could be a candidate frequency for evolved functionalities in brain.
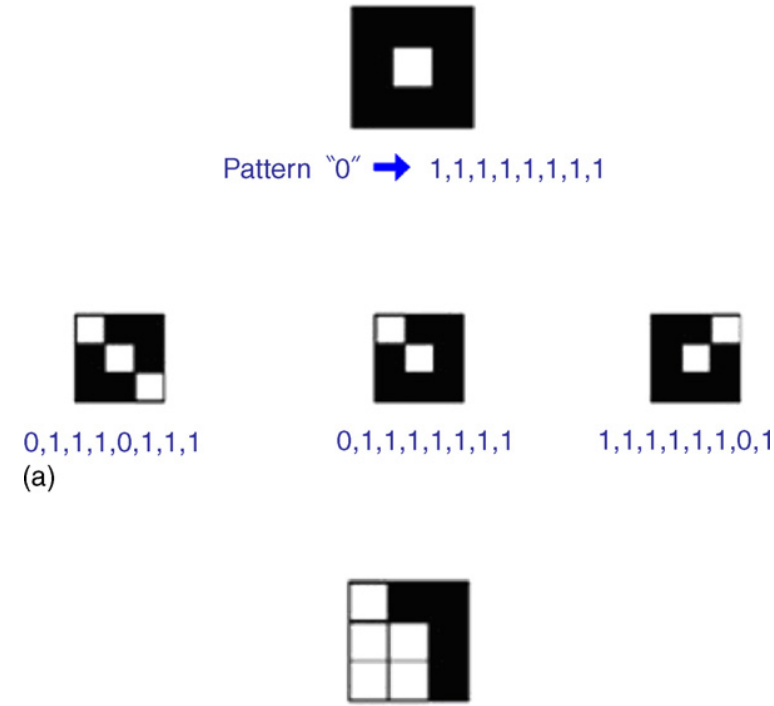

Pattern " 1 " $\Rightarrow 0,0,0,0,1,1,1,1$

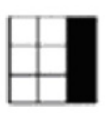

$0,0,0,0,1,1,1,0$

(b)

Fig. 9. (a) "0" Bitmap and "0 with noise" bitmaps submitted to the hybrid network. Each black square in the bitmap corresponds to a $35 \mathrm{mV}$ voltage and is injected to one of the eight electrodes. As the central bit is always white, it is not referred to any electrode. (b) "1" Bitmap and "1 with noise" bitmaps submitted to the hybrid network.

Thus we acquired the signals with a $10 \mathrm{kHz}$ sampling rate for each channel, then we applied a $40 \mathrm{~Hz}$ low pass filter offline before the signal analysis, in order to highlight possible correlations between signals at the frequencies in the range between 0 and $40 \mathrm{~Hz}$. We considered frequencies lower than $40 \mathrm{~Hz}$, as in our experience and in other authors electrical correlated activity has been verified also at $20 \mathrm{~Hz}$ or less (Joliot et al., 1994).

In order to allow the choice of different " 0 " and " 1 " patterns, pure or affected by noise, we developed a software that interfaces with the acquisition card and allows drawing the desired bitmaps to be sent to the network. The software also allows setting the number of cycles of the chosen sequence of patterns, the waiting time between sequences, the number of iterations, and the name of the file that will be recorded.

The output signals have been analyzed to evaluate the possibility of organized reaction by the natural neurons.

Although stem cells show the essential properties of functional CNS neurons, their detailed behavior is still in course of study (Magistretti et al., 1996; McKay, 1997; Gritti et al., 2000). For this reason our analysis does not search for known features and artefacts in signals, but utilizes tools able to mea- 
sure the degree of organization of signals during and after training.

To this purpose after the experiment the output signals have been analyzed using the recurrence quantification analysis (Zbilut and Webber, 1992; Zbilut et al., 2002).

\subsection{Recurrence quantification analysis}

It has been proven that a topologically equivalent picture of a multivariable dynamical system behavior can be created by using the time series of a single observable variable (Takens, 1981). The basic idea is that the effect of all the other (unobserved) variables is already reflected in the series of the observed output. Thus a one-dimensional time series from a data file is expanded into a higher-dimensional space using a technique called "delay-time embedding", that recreates a phase space portrait of the dynamical system under study from a single (scalar) time series.

To expand a one-dimensional signal into an $m$-dimensional phase space, one substitutes each observation in the original signal $X(t)$ with the vector:

$y_{i}=\left\{x_{i}, x_{i-d}, x_{i-2 d}, \ldots, x_{i-(m-1) d}\right\}$,

where $i$ is the time index, $m$ the embedding dimension, and $d$ is the time delay.

As a result, we have a series of vectors:

$Y=\{y(1), y(2), y(3), \ldots, y(N-(m-1) d)\}$,

where $N$ is the length of the original series.

Each unknown state $S_{t}$ at time $t$ is approximated by a vector of delayed coordinates $Y_{t}=\left\{x_{t}, x_{t-d}, x_{t-2 d}, \ldots, x_{t-(m-1) d}\right\}$.

Recurrence quantification analysis (RQA) is a new quantitative tool that can be applied to time series reconstructed with delay-time embedding. RQA is independent of data set size, data stationarity, and assumptions on statistical distributions of data.

RQA gives a local view of the series behavior, because it analyzes distances of pairs of points, not a distribution of distances. Therefore, unlike autocorrelation, RQA is able to analyze fast transients and to localize in time the features of a dynamical variation: for this reasons RQA is ideally suited for physiological systems. Once the dynamical system is reconstructed in a manner outlined above, a "recurrent plot" can be used to show which vectors in the reconstructed space are close and far from each other.

The recurrent plots show how the vectors in the reconstructed space are near or distant each other. They preserve the temporal dependence in the time series, in addition to the spatial dependence.

More specifically, the plot represents the (Euclidean) distances between all pairs of vectors and codes them as colors. Essentially, a recurrent plot is a color-coded matrix, where each $[i][j]$ th entry is calculated as the distance between vectors $Y_{i}$ and $Y_{j}$ in the reconstructed series. After the distances between all vectors are calculated, they are mapped to colors from the pre-defined color map and are displayed as colored pix- els in their corresponding places, as in the geographical relief maps.

The observation of recurrent points consecutive in time (forming lines parallel to the main diagonal) is an important signature of deterministic structure. In fact the length of the longest diagonal (recurrent) line accurately corresponds to the value of the maximum Lyapounov exponent of the series. The Lyapounov exponent is a measure of chaoticity, quantifying the mean rate of divergence of neighbouring trajectories along various directions in the phase space of an embedded time series. Time series of chaotic systems have a positive maximum Lyapounov exponent.

For random signals, the uniform distribution of colors over the entire RP is expected. The more deterministic the signal, the more structured the recurrent plot.

The recurrent plots version we adopted (Kononov, 1996) calculates the Euclidean distances between all the vector pairs and translates them into colour bands.

Hot colours (yellow, red, orange) are associated to short distances between vectors, cold colours (blue, black) show long distances. Signals repeating fixed distances between vectors are organized, signals without repeating distances are not. In this way we obtain uniform colour distribution for random signals, but the more deterministic and self-similar is the signal, the more structured is the plot.

The RQA analysis in our experiments has the only aim to show the behavior variation of the signals emitted by the biological network after the injection of the organized stimuli.

The signals are elaborated by the RQA algorithm following the artificial neural networks paradigm, i.e., handling them as a unique signal where the sample coming from the entire output channel follow one upon the other.

\subsection{ITSOM analysis}

In order to decode the output signals of the biological network we put them in cascade with an artificial neural network.

The model of ANN, a novel architecture called ITSOM (inductive tracing self organizing map), was selected considering that a self-organizing architecture was necessary, as we had not a set of examples to train it. Secondly, the selected ANN was tested in the past with electrophysiological signals (Pizzi et al., 2002), correctly showing their organized structures.

Finally, the extremely low processing time makes this model very effective in case of real-time applications that are in our future prospects.

As we have seen in Section 2.3.1, the main SOM feature is to identify a winning neuron that should classify the input stream. But two main reasons exist that limit the SOM's performances in case of strictly non-linear and time-variant input.

The first reason is that if the input topology is too tangled, the competitive layer is not able to unfold itself enough to simulate the input topology.

The second reason concerns the SOM's convergence conditions that exist but are not easily verifiable. Due to the nature of the SOM's output (non-homologous to the input), it is not 
possible to settle either a network error for each epoch, or the number of epochs after that the network training has to be stopped.

Nevertheless, as in many cases even after several thousands of epochs the convergence was not reached, the processing time was verified to become too long for a real-time application.

Another problem of the SOM, typical of any clustering algorithm, is the lack of output explication. Once a classification is obtained, the user must analyze it, comparing it to the input values in order to extrapolate a significant output.

Thus we proposed a structural modification of the SOM called inductive tracing self-organizing map (ITSOM). The dynamical properties of artificial neural networks and of the SOM in particular are well known (Rosen, 1970; Pineda, 1988; Jeffries, 1991; Ritter and Schulten, 1986, 1988; Ermentrout, 1992).

During simulations carried on with the SOM algorithm we have observed that, even if the winning weights may vary at any presentation epoch, their temporal sequence tends to repeat itself (Pizzi, 1997).

A deeper analysis has shown that such a sequence, provided to keep the learning rates steady (instead of gradually decreasing them), constitutes chaotic attractors that repeat "nearly" exactly in time with the epochs succeeding, and that, once codified by the network, univocally characterize the input element that has determined them.

Actually the SOM learning rule, as explained above, makes it possible for the winning weight to represent an approximation of the input value. In this way at every epoch the new winning weight, together with the previous winner, constitutes a second-order approximation of the input value. At the $n$-th epoch, the set of $n$ winning weights represents an $n$ th-order approximation of the input value.

In this way, due to the countless variety of possible combinations among winning neurons, the configurations allow to finely determine the correct value, even in the case of tangled input topologies, despite of the small number of competitive neurons and their linear topology.

In the following step the network performs a real induction process, because after a many-to-few vector quantization from the input to the weight layer (to be precise, to the chaotic configurations of winning weights), a few-to-many procedure is performed from the chaotic configurations corresponding to the input set (Fig. 10) codified by the network.

It should be stressed that the ITSOMs crucial feature is that the network does not need to be brought to convergence, as the cyclic configurations stabilize their structure within a small number of epochs, then keep it steady through time.

After interrupting the network-processing phase, an algorithm is needed that codifies the obtained chaotic configurations into a small set of outputs.

The algorithm that has shown best performances and computational load among the tested pattern recognition algorithms is based on a $z$-score calculus.

The cumulative scores related to each input have been normalized following the distribution of the standardized variable

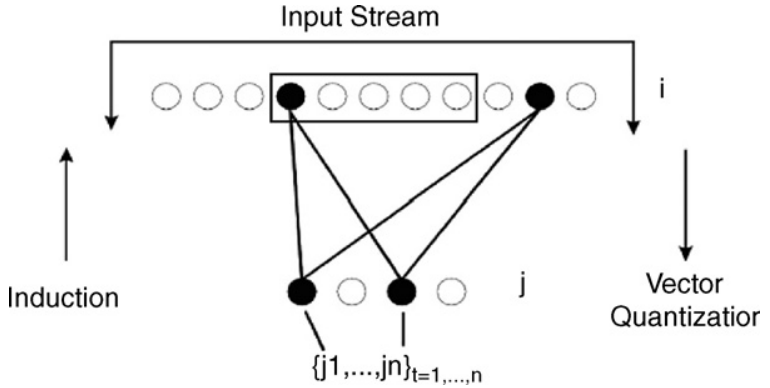

Fig. 10. The ITSOM network selects in the competitive layer a series of winning neurons in time. The set of winning neurons identifies the input pattern.

$z$ given by:

$z=\frac{(x-\mu)}{\sigma}$

where $\mu$ is the average of the scores on all the competitive layer weights and $\sigma$ is the root mean squared deviation. Once fixed a threshold $0<\tau \leq 1$, we have put

$z=1$ for $z>\tau, \quad z=0$ for $z \leq \tau$.

In this way every winning configuration is represented by a binary number with as many 1's and 0's as many the competitive layer weights.

Then the task of comparing these binary numbers is straightforward.

It has been verified that the $\tau$ threshold size is not critical: fixing it to 0.5 we have obtained the best results with any input stream.

The $z$-score method has shown to be steady with regard of the performances, and computationally not expensive, being linear in the number of the competitive layer weights.

But it is worth emphasizing that the $z$-score algorithm allows the network to reach its best performances in a very small number of epochs (often less then 15).

This allows the network to complete its work within a negligible time, and to actually assert the possibility of a real-time processing.

The good performances of this network have been tested in the classification of neurological diseases (Pizzi et al., 1997), for equalization and demodulation of GSM signals (Favalli et al., 1996), for image classification (Pizzi et al., 1998) and for EEG analysis (Pizzi et al., 2002).

The small computational load is an element that makes the ITSOM suitable for a possible hardware implementation (Chen et al., 1992; Choi et al., 1993).

\section{Results}

During the experiments cells are kept in a controlled environment a $37^{\circ} \mathrm{C}$. At the end of the experiment the neurons are alive and maintain their functionalities. 


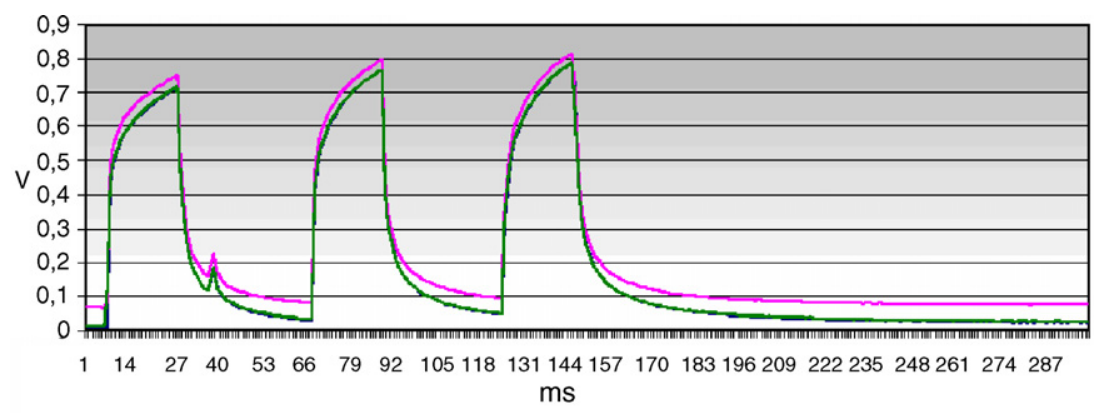

Fig. 11. Culture liquid output during stimulation with "0" patterns: conductor-like behavior.

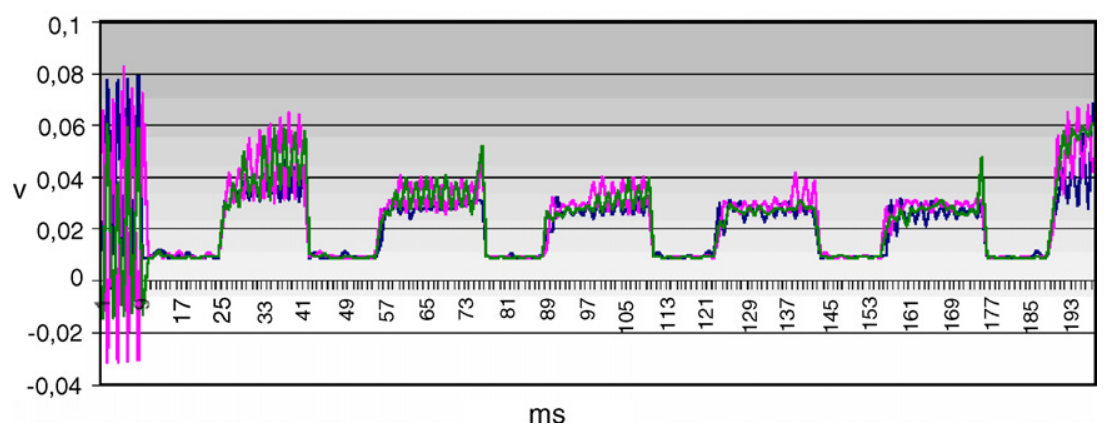

Fig. 12. Network output during stimulation with " 0 " patterns: the signals show a low voltage $(10 \mathrm{mV})$ behavior during the $35 \mathrm{mV}$ stimulation.

In order to check if the signals received by the acquisition device were actually coming from neurons, we measured the reactions of the only culture liquid with fibroblasts (Fig. 11), comparing them with those coming from the cells (Fig. 12).

We iterated the experiment with different stimulation lengths, ranging from 1.25 to $25 \mathrm{~ms}$.

It is evident that the network reacts to the " 0 " pattern, constituted by the highest voltage (11111111), emitting the lowest voltages. The culture liquid, instead, reacts to the "0" pattern with a high voltage, much higher than the neural voltages, as correctly expected by a conductive medium.

In Fig. 13 is depicted the reaction of the Kohonen network after stimulation with " 0 " patterns, pure and affected by noise (first and third circle from the left), and with " 1 " patterns (second and fourth circle), pure and affected by noise.

Similar effects have been shown by the Hopfield network. At the end of the experiment we measured the network output in order to evaluate if the neurons had "stored" information in some way. Differently from the only culture liquid, that shows the same behavior before and after experiment, the network output retains different voltages.
The analysis of our data using the RQA method leads to interesting results. Signals coming from similar bitmaps gave rise to similar recurrent plots.

Moreover, the following figures show the selforganization of a single output channel before stimulation, during the training, during the testing phase, and after stimulation.

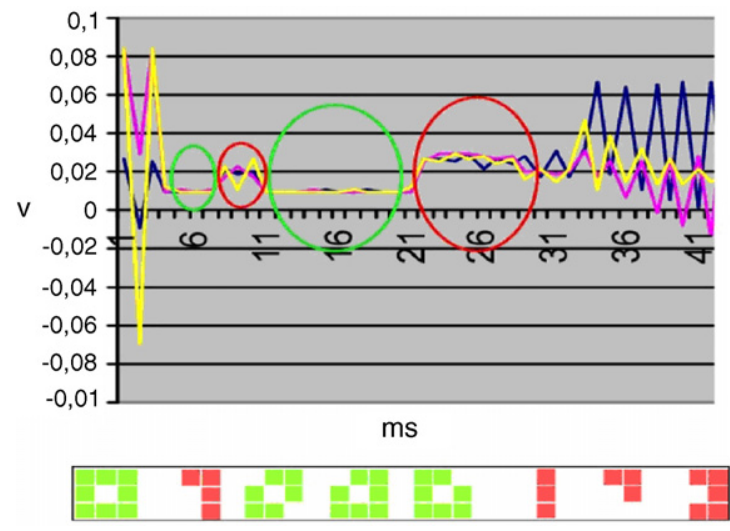

Fig. 13. Kohonen network output during training: different voltages for different patterns. The red circles highlight the reaction of neurons to " 1 " patterns, the green circles the reaction to " 0 " patterns. 


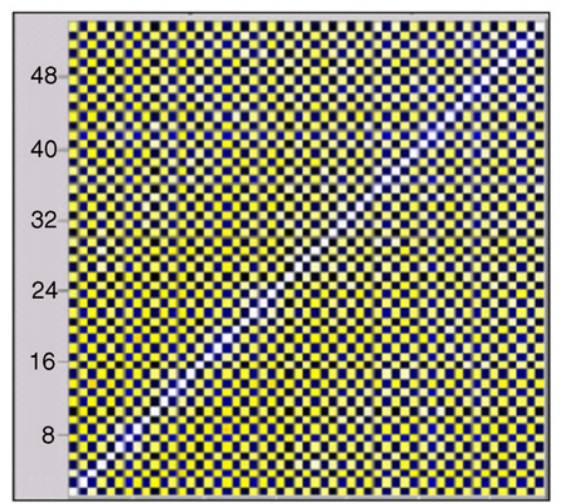

(a)

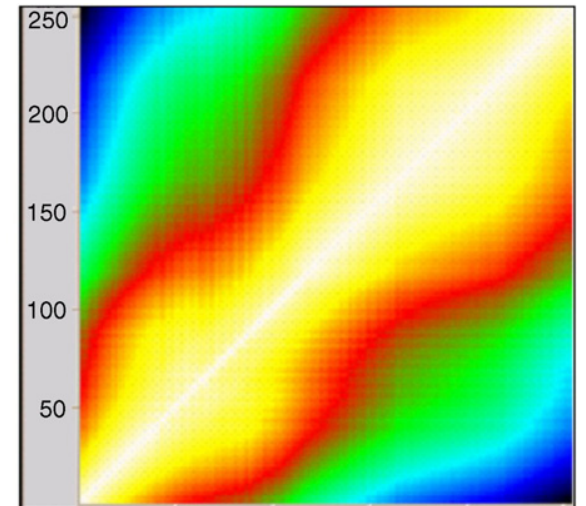

(b)

Fig. 14. RQA plot of the Kohonen network before stimulation ((a) no organization) and after training ((b) high organization). The plots represent a portion of the whole signal, and the $x-y$ axes indicate the sequential number of the considered samples.

Fig. 14a shows one output channel of the Kohonen network before stimulation. Colours are cold and unstructured, showing lack of self-organization.

The training phase shows a change in the structure. The recurrent plot of the channel during the testing phase shows wide uniform hot colour bands corresponding to a high organization. Fig. 14b is the plot of the output channel after the end of stimulations. In this case the uniform bands further widen, showing that the signal remains self-organized in time.

This analysis shows that introduction of organized stimuli modifies the network structure and increases the information content even after the end of stimulation, suggesting a form of learning and memorization.

We applied the same procedure to the output signals coming from the Hopfield network.

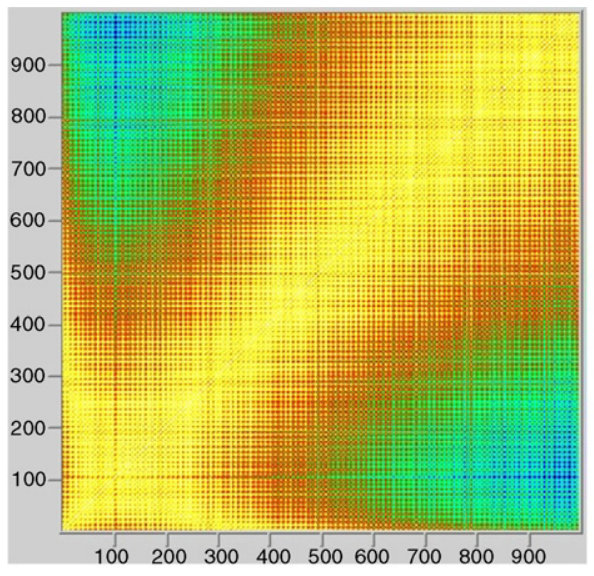

(a)
Fig. 15a shows one channel after stimulation with the "0" pattern: we see wide organized bands with peculiar features, different from the other channels. Fig. 15b shows the same channel after stimulation with " 1 " pattern.

This analysis shows that the network behaves differently depending on the input signal and on the different channels.

The RQA analysis had the only aim to show the different behavior of the signals emitted by the biological network before and after the injection of organized stimuli. The analysis shows that the cells maintain after the end of stimulation an organization state that was not present before the stimulation: this is also what happens in the structure of an artificial neural network after learning.

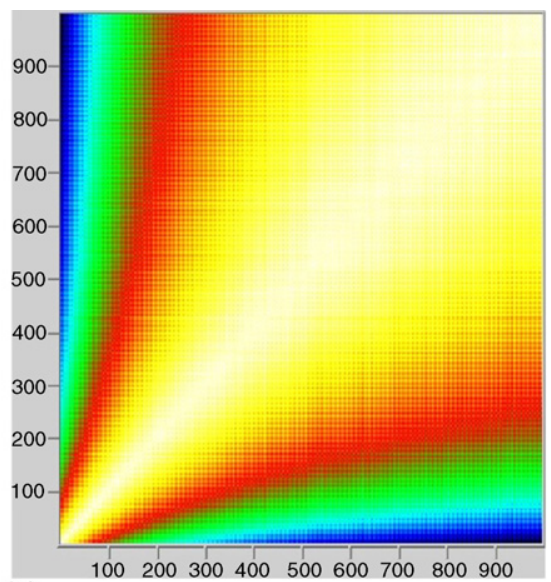

(b)

Fig. 15. RQA plot of one channel of the Hopfield network after stimulation with "0" patterns (a) and " 1 " patterns (b): different kinds of organized structures. 
Table 1

$z$-Score code of some " 0 " patterns: the ITSOM network assigns the same code to the electrical reaction to similar patterns

\begin{tabular}{|c|c|c|c|c|c|c|c|c|c|c|c|c|c|c|c|c|c|}
\hline “0” Pattern & 1 & 0 & 0 & 0 & 1 & 1 & 0 & 0 & 1 & 1 & 0 & 0 & 1 & 1 & 0 & 0 & 1 \\
\hline " 0 " With noise, type 1 & 1 & 0 & 0 & 0 & 1 & 1 & 0 & 0 & 1 & 1 & 0 & 0 & 1 & 1 & 0 & 0 & 1 \\
\hline "0" With noise, type 2 & 1 & 0 & 0 & 0 & 1 & 1 & 0 & 0 & 1 & 1 & 0 & 0 & 1 & 1 & 0 & 0 & 1 \\
\hline "0" With noise, type 3 & 1 & 0 & 0 & 0 & 1 & 1 & 0 & 0 & 1 & 1 & 0 & 0 & 1 & 1 & 0 & 0 & 1 \\
\hline "0" With noise, type 4 & 1 & 0 & 0 & 0 & 1 & 1 & 0 & 0 & 1 & 1 & 0 & 0 & 1 & 1 & 0 & 0 & 1 \\
\hline "0" With noise, type 5 & 1 & 0 & 0 & 0 & 1 & 1 & 0 & 0 & 1 & 1 & 0 & 0 & 1 & 1 & 0 & 0 & 1 \\
\hline " 0 " With noise, type 6 & 1 & 0 & 0 & 0 & 1 & 1 & 0 & 0 & 1 & 1 & 0 & 0 & 1 & 1 & 0 & 0 & 1 \\
\hline " 0 " With noise, type 7 & 1 & 0 & 0 & 0 & 1 & 1 & 0 & 0 & 1 & 1 & 0 & 0 & 1 & 1 & 0 & 0 & 1 \\
\hline " 0 " With noise, type 8 & 1 & 0 & 0 & 0 & 1 & 1 & 0 & 0 & 1 & 1 & 0 & 0 & 1 & 1 & 0 & 0 & 1 \\
\hline
\end{tabular}

In order to confirm the results drawn by means of the RQA analysis and to decode the information content of the output signals, we used the ITSOM artificial neural network described in Section 2.6.

The ITSOM network has been applied to signals coming from outputs of different bitmaps.

We used a network with 250 input neurons and 15 competitive neurons. Due to the organized content of the signals, the chaotic attractors repeated themselves after around 20 epochs.

As expected after the RQA analysis, the network has discriminated different bitmaps with different series of winning neurons, whereas similar bitmaps have shown an identical series of winning neurons.

In Table 1 we represented the codes generated by the ITSOM elaborating the signals emitted by the cells after stimulating them with the " 0 " pattern (row 1) and other " 0 " patterns affected by noise, as in Fig. 8 . The table shows that the ITSOM generated the same code for all the patterns.

In this example the artificial neural network, analyzing the sequence of samples coming from the eight electrodes after a " 0 " stimulation, cycles 20 times.

In this case, during competition the networks "won" five times in node 1 , zero times in node 2,0 in node 3,3 in node 4,3 in node 5 and so on. After the $z$-score procedure we obtained the code as in the first row of Table 1. With the second pattern " 0 with noise", the network "won" just four times in node 1,0 in node 2,0 in node 3,4 in node 4 and so on, but the $z$-score procedure provided the same code.

\section{Discussion and conclusions}

After our analysis of the output signals of the networks we can reasonably affirm that the networks show an organized behavior after the stimulation with patterns, and they are able to answer selectively to different patterns. The signal behavior changes depending on the network channels, and similar patterns give rise to similar answers.

Thus we can say that the networks have shown a form of selective coding, highlighting a strong selforganization as a reply to stimulation, that persists for a long time after the stimulation bursts.

Moreover, despite the lack of a detailed neurophysiological interpretation of the signal tracing, the ITSOM network has allowed to distinguish the different information contents of the signals. We could show that similar patterns give rise to output signals containing similar chaotic attractors that have been codified, whereas different patterns lead to attractors corresponding to different codes.

Being able to discriminate and interpret the information content of the biological network, we are planning to use in the future these outputs in several ways.

In fact, the aim of this kind of research is on one hand to improve the knowledge of the neurophysiological learning and memory functionalities; on the other hand it would be possible to evaluate the feasibility of a hybrid electronic-biological device, conceiving the possibility of biological computation, or of non-invasive neurological prostheses, able to improve or substitute for damaged nervous functionalities.

The culture method we adopted revealed to be suitable for our experiments, ensuring the necessary survival of cells, but at the moment neurons do not remain alive more than about 2 months. Nonetheless a better culture method and more suitable MEA supports are under study and a future improvement of the culture duration is expected.

Another problem that should be solved is the increase in complexity of the artificial connection on the MEAs in order to pass from prototype patterns to complex patterns.

By increasing the number of connections and of input electrodes, the acquisition card will share the sampling rate into more channels, diminishing its perfor- 
mances. At the moment we have already acquired a new more powerful acquisition card (National Instruments PXI6251), and we started experiments with six more complex input bitmaps, maintaining the same number of input channels and delivering the patterns to the hybrid network eight bits at a time, following a well-known artificial neural networks technique. Analyses of the results are under way, but preliminary studies show the same recognition capabilities as those described in this paper. Another improvement will be the implementation of a printed circuit board controller instead of the current wired hardware, in order to ensure robustness and flexibility.

As we verified the possibility to codify the neuron output, we are developing the interface between the codified output and a simple actuator (a minirobot), with the purpose to experiment a complete chain perception (input patterns) - hybrid neural network - action: this is possible due to the ITSOM real-time coding of the output stream.

Off-line experiments have been already carried out and the whole real-time system is expected in the next few months.

\section{Acknowledgements}

We are strongly indebted to Prof. G. Degli Antoni (University of Milan) for his valuable suggestions and encouragement, to Dr. Francesca Gregori for her substantial contribution and to ST Microelectronics for the important support.

\section{References}

Adrian, E.D., 1950. The electrical activity of the mammalian olfactory bulb. Electroencephalogr. Clin. Neurophysiol. 2, 377-388.

Akin, T., Najafi, K., Smoke, R.H., Bradley, R.M., 1994. A micromachined silicon electrode for nerve regeneration applications. IEEE Trans. Biomed. Eng. 41, 305-313.

Bels, B., Fromherz, P., 2002. Transistor array with an organotypic brain slice: field potential records and synaptic currents. Eur. J. Neurosci. $15,999-1005$.

Bonifazi, P., Fromherz, P., 2002. Silicon chip for electronic communication between nerve cells by non-invasive interfacing and analog-digital processing. Adv. Mater. 17.

Borkholder, D.A., Bao, J., Maluf, N.I., Perl, E.R., Kovacs, G.T., 1997. Microelectrode arrays for stimulation of neural slice preparations. J. Neurosci. Methods 7, 61-66.

Bouyer, J.J., Montaron, M.F., Vahnee, J.M., Albert, M.P., Rougeul, A., 1987. Anatomical localization of cortical beta rhythms in cat. Neuroscience 22, 863-869.

Bove, M., Martinoia, S., Grattarola, M., Ricci, D., 1996. The neurontransistor junction: linking equivalent electric circuit models to microscopic descriptions. Thin Solid Films 285, 772-775.
Bragin, A., Jandф, G., Ndasdy, Z., Hetke, J., Wise, K., Buzs ki, G., 1995. Gamma $(40-100 \mathrm{~Hz})$ oscillation in the hippocampus of the behaving rat. J. Neurosci. 15, 47-60.

Braun, D., Fromherz, P., 2001. Fast voltage transients in capacitive silicon-to-cell stimulation detected with a luminescent molecular electronic probe. Phys. Rev. Lett. 13 (86), 2905-2908.

Breckenridge, L.J., Wilson, R.J.A., Connolly, P., Curtis, A.S.G., Dow, J.A.T., Blackshaw, S.E., Wilkinson, C.D.W., 1995. Advantages of using microfabricated extracellular electrodes for in vitro neuronal recording. J. Neurosci. Res. 42, 266-276.

Canepari, M., Bove, M., Mueda, E., Cappello, M., Kawana, A., 1997. Experimental analysis of neural dynamics in cultured cortical networks and transitions between different patterns of activity. Biol. Cybernet. 77, 153-162.

Carmena, J.M., Lebedev, M.A., Crist, R.E., O’Doherty, J.E., Santucci, D.M., Dimitrov, D.F., Patil, P.G., Henriquez, C.S., 2003. Learning to control a brain-machine interface for reaching and grasping by primates. M.A.L. PLoS 1, 193-208.

Chen, O., Sheu, B., Fang, W., 1992. Image compression on VLSI neural-based vector quantizer. Inf. Process. Manage. 28, 6.

Choi, J., Bang, S.H., Sheu, B.J., 1993. A Programmable analog VLSI neural network processor for communication receivers. IEEE Trans. Neural Network 4, 3.

Chrobak, J.J., Buzsaki, G., 1998. Gamma oscillations in the entorhinal cortex of the freely behaving rat. J. Neurosci. 18, 388-398.

De Marse, T.B., Wagenaar, D.A., Potter, S.M., 2002. The Neurallycontrolled Artificial Animal: A Neural Computer Interface Between Cultured Neural Networks and a Robotic Body. SFN 2002, Orlando, Florida.

Dickson, C.T., Biella, G., de Curtis, M., 2000. Evidence for spatial modules mediated by temporal synchronization of carbacholinduced gamma rhythm in medial entorhinal cortex. J. Neurosci. 20, 7846-7854.

Durbin, R., Mitchinson, G., 1990. A dimension reduction framework for understanding cortical maps. Nature 343, 644-647.

Eckhorn, R., Bauer, R., Jordan, W., Brosch, M., Kruse, W., Munk, M., Reitboeck, H.J., 1998. Coherent oscillations: a mechanism of feature linking in the visual cortex? Multiple electrode and correlation analyses in the cat. Biol. Cybern. 60, 121-130.

Eckhorn, R., Reitboeck, H.J., Arndt, M., Dicke, P., 1990. Feature linking via synchronization among distributed assemblies: simulations of results from cat visual cortex. Neural Comput. 2, 293-307.

Eeckman, F.H., Freeman, W.J., 1990. Correlations between unit firing and EEG in the rat olfactory system. Brain Res. 528, 238-244.

Egert, U., Schlosshauer, B., Fennrich, S., Nisch, W., Fejtl, M., Knott, T., Müller, T., Hammerle, H., 1988. A novel organotypic long-term culture of the rat hippocampus on substrate-integrated microelectrode arrays. Brain Resour. Protoc. 2, 229-242.

Engel, A.K., König, P., Singer, W., 1991a. Direct physiological evidence for scene segmentation by temporal coding. Proc. Natl. Acad. Sci. USA 88, 9136-9140.

Engel, A.K., König, P., Kreiter, A.K., Singer, W., 1991b. Interhemispheric synchronization of oscillatory neuronal responses in cat visual cortex. Science 252, 1177-1179.

Ermentrout, B., 1992. Complex dynamics in WTA neural networks with slow inhibition. Neural Networks 5, 415-431.

Favalli, L., Mecocci, A., Pizzi, R., 1996. A soft receiver using recurrent networks. In: Proc. EUSIPCO96, VII European Signal Processing Conference, Trieste.

Freeman, W.J., 1994. Role of chaotic dynamics in neural plasticity. In: Van Pelt, J., Lopes da Silva, F.H. (Eds.), The Self-organizing Brain: from Growth Cones to Functional Networks. Elsevier. 
Freeman, W.J., van Dijk, B.W., 1987. Spatial patterns of visual cortical fast EEG during conditioned reflex in a rhesus monkey. Brain Res. 422, 267-276.

Freeman, W.J., 1987. Relation of olfactory EEG on behaviour: time series analysis. Behav. Neurosci. 100.

Fromherz, P., 2002. Electrical interfacing of nerve cells and semiconductor chips. Chem. Phys. Chem. 3, 276-284.

Fromherz, P., Muller, C.O., Weis, R., 1993. Neuron-transistor: electrical transfer function measured by the Patch-Clamp technique. Phys. Rev. Lett. 71, 4079-4082.

Fromherz, P., Schaden, H., 1994. Defined neuronal arborisations by guided outgrowth of leech neurons in culture. Eur. J. Neurosci. 6.

Fromherz, P., Offenhäusser, A., Vetter, T., Weis, J., 1991. A neuronsilicon-junction: a Retzius-cell of the leech on an insulated-gate field-effect transistor. Science 252, 1290-1293.

Galambos, R., Makeig, S., Talmachoff, P.J., 1981. A 40-Hz auditory potential recorded from the human scalp. Proc. Natl. Acad. Sci. USA 78, 2643-2647.

Garcia, P.S., Calabrese, R.L., DeWeerth, S.P., Ditto, W., 2003. Simple arithmetic with firing rate encoding in leech neurons: simulation and experiment. In: Proceedings of the XXVI Australian Computer Science Conference, vol. 16, Adelaide, pp. 55-60.

Gray, C.M., König, P., Engel, A.K., Singer, W., 1989. Oscillatory responses in cat visual cortex exhibit inter-columnar synchronization which reflects global stimulus properties. Nature 338, 334-337.

Gritti, A., Rosati, B., Lecchi, M., Vescovi, A.L., Wanke, E., 2000. Excitable properties in astrocytes derived from human embryonic CNS stem cells. Eur. J. Neurosci. 12, 3549-3559.

Gritti, A., Galli, R., Vescovi, A.L., 2001. In: Federoff (Ed.), Culture of Stem Cell of Central Nervous System. Humana Press III, pp. 173-197.

Haken, H., 1991. Synergetic Computers and Cognition (A Top-down Approach to Neural Nets). Springer-Verlag.

Haykin, S., 1994. Neural Networks (A Comprehensive Foundation). MacMillan Coll. Pub.

Hopfield, J.J., 1984. Neural networks and physical systems with emergent collective computational abilities. Proc. Natl. Acad. Sci. USA, 81.

Hubel, D.H., Wiesel, T.N., 1962. Receptive fields, binocular interaction and functional architecture in the cat's visual cortex. J. Physiol. London 160, 106-154.

Hubel, D.H., Wiesel, T.N., 1968. Receptive fields and functional architecture of monkey striate cortex. J. Physiol. 195, 215-243.

Hubel, D.H., Wiesel, T.N., 1974. Sequence regularity and geometry of orientation columns in the monkey striate cortex. J. Comp. Neurol. $158,267-293$.

Jeffries, C., 1991. Code Recognition and Set Selection with Neural Networks. Birkhauser Boston.

Jenkner, M., Fromherz, P., 1997. Bistability of membrane conductance in cell adhesion observed in a neuron transistor. Phys. Rev. Lett. 79, 4705-4708.

Jenkner, M., Muller, B., Fromherz, P., 2001. Interfacing a silicon chip to pairs of snail neurons connected by electrical synapses. Biol. Cybernet. 84, 239-249.

Jimbo, Y., Robinson, H.P.C., 2000. Propagation of spontaneous synchronized activity in cortical slice cultures recorded by planar electrode arrays. Bioelectrochemistry 5, 107-115.

Joliot, M., Ribary, U., Llinas, R., 1994. Human oscillatory brain activity near $40 \mathrm{~Hz}$ coexists with cognitive temporal binding. Proc. Natl. Acad. Sci USA 91, 11748-11751.
Knudsen, E., du Lac, S., Esterly, S.D., 1987. Computational maps in the brain. Ann. Rev. Neurosci., 10-41.

Kohonen, T., 1990. Self-Organisation and Association Memory. Springer-Verlag.

Kononov, E., 1996. http://home.netcom.com/ eugenek/download.html.

Kulli, J., Koch, C., 1991. Does anesthesia cause loss of consciousness. Trends Neurosci. 14, 6-10.

Lindner, J.F., Ditto, W., 1996. Exploring the nonlinear dynamics of a physiologically viable model neuron. AIP Conf. Proc. 1, 375-385.

Llinas, R., Ribary, U., 1993. Coherent 40-Hz oscillation characterizes dream state in humans. Proc. Natl. Acad. Sci. USA 90, 2078-2081.

Madler, C., Keller, I., Schwender, D., Poppel, E., 1991. Sensory information processing during general anaesthesia: effect of isoflurane on auditory evoked neuronal oscillations. Br. J. Anaesth. 66, 81-87.

Magistretti, J., Mantegazza, M., Guatteo, E., Wanke, E., 1996. Action potential recorded with patch clamp amplifiers: are they genuine? Trends Neurosci. 19, 531-534.

Maher, M.P., Pine, J., Wright, J., Tai, Y.C., 1999. The neurochip: a new multielectrode device for stimulating and recording from cultured neurons. Neurosci. Methods 87, 45-56.

Mc Culloch, W., Pitt, W., 1943. A logical calculus of the ideas immanent in nervous activity. Bull. Math. Biophys. 5, 115-133.

McKay, R.D.G., 1997. Stem cells in the central nervous system. Science 276, 66-71.

Menon, V., Freeman, W.J., 1996. Spatio-temporal correlations in human gamma band electrocorticograms. Electroencephalogr. Clin. Neurophys. 98, 89-102.

Mitner, W.H.R., Braun, C., Arnold, M., Witte, H., Taub, E., 1999. Coherence of gamma-band EEG activity as a basis for associative learning. Nature 397, 434-436.

Mountcastle, V.B., 1957. Modality and topographic properties of single neurons of cat's somatic sensory cortex. J. Neurophysiol. 20, 408-434.

Murthy, V.N., Fetz, E.E., 1992. Coherent 25-35-Hz oscillations in the sensorimotor cortex of awake behaving monkeys. Proc. Natl. Acad. Sci. USA 89, 5670-5674.

Pantev, C., Makeig, S., Hoke, M., Galambos, R., Hampson, S., Gallen, C., 1991. Human auditory evoked gamma-band magnetic fields. Proc. Natl. Acad. Sci. USA 88, 8996-9000.

Pfurtscheller, G., Flotzinger, D., Neuper, C., 1994. Differentiation between finger, toe and tongue movement in man based on $40 \mathrm{~Hz}$ EEG. Electroencephalogr. Clin. Neurophysiol. 90, 456-460.

Pineda, F.J., 1988. Dynamics and architectures for neural computation. J. Complex. 4, 216-245.

Pizzi, R., 1997. Theory of Dynamical Neural Systems with application to Telecommunications, PhD Dissertation, University of Pavia.

Pizzi, R., de Curtis, M., Dickson, C., 2002. Evidence of chaotic attractors in cortical fast oscillations tested by an artificial neural network. In: Kacprzyk, J. (Ed.), Advances in Soft Computing. Physica Verlag.

Pizzi, R., Reni, G., Sicurello, F., Varini, G., 1997. Comparison between clustering algorithms and neural networks for the classification of neurological diseases. In: Proc. Softstat 97, Heidelberg.

Pizzi, R., Sicurello, F., Varini, G., 1998. Development of an inductive self-organizing network for the real-time segmentation of diagnostic images. In: Proceeding of the Third International Conference of Neural Networks and Expert Systems in Medicine and Healthcare, Pisa, pp. 44-50.

Potter, S.M., 2001. Distributed processing in cultured neuronal networks. In: Nicolelis (Ed.), Progress in Brain Research, M.A.L. Elsevier Science B.V. 
Reger, B., Fleming, K.M., Sanguineti, V., Simon Alford, S., MussaIvaldi, F.A., 2000. Connecting brains to robots: an artificial body for studying the computational properties of neural tissues. Artif. Life 6, 307-324.

Ritter, H., Schulten, K., 1986. On the stationary state of Kohonen's self-organizing sensory mapping. Biol. Cybernet. 54, 99106.

Ritter, H., Schulten, K., 1988. Convergence properties of Kohonen's topology conserving maps: fluctuations, stability, and dimension selection. Biol. Cybernet. 60, 59-71.

Rosen, R., 1970. Dynamical System Theory in Biology. Stability Theory and its applications, vol. 1. Wiley Interscience.

Rosenblatt, F., 1958. The perceptron: a probabilistic model for information storage and organization in the brain. Psychol. Rev. 65, 386-408.

Rumelhart, D.E., Mcclelland, J.L., 1986. Parallel Distributed Processing. MIT Press, Cambridge.

Saarinen, J., Kohonen, T., 1985. Self-organized formation of colour maps in a model cortex. Perception 14, 711-719.

Sanes, J.N., Donoghue, J.P., 1993. Oscillations in local field potentials of the primate motor cortex during voluntary movement. Proc. Natl. Acad. Sci. USA 90, 4470-4474.

Schatzthauer, R., Fromherz, P., 1998. Neuron-silicon junction with voltage gated ionic currents. Eur. J. Neurosci. 10, 1956-1962.

Schiff, S.J., Jerger, K., Duong, D.H., Chang, T., Spano, M.L., Ditto, W., 1994. Controlling chaos in the brain. Nature 8, 25.

School, M., Sprössler, C., Denyer, M., Krause, M., Nakajima, K., Maelicke, A., Knoll, W., Offenhäusser, 2000. Ordered networks of rat hippocampal neurons attached to silicon oxide surfaces. Neurosci. Methods 104, 65-75.

Singer, W., Gray, C.M., 1995. Visual feature integration and the temporal correlation hypothesis. Ann. Rev. Neurosci. 18, 555586.

Song, H., Stevens, C.F., Gage, F.H., 2002. Neural stem cells from adult hippocampus develop essential properties of functional CNS neurons. Nature Neurosci. 5, 5.
Stumpf, C., 1965. The fast component in the electrical activity of rabbit's hippocampus. Electroencephalogr. Clin. Neurophysiol. 18, 477-486.

Takens, F., 1981. Detecting strange actractors in turbulence. In: Rand, D.A., Young, L.S. (Eds.), Dynamical Systems and TurbulenceLecture Notes in Mathematics, vol. 898. Springer-Verlag.

Tank, D.W., Hopfield, J.J., 1989. Neural Architecture and Biophysics for Sequence Recognition in Neural Models of Plasticity. Academic Press.

Tiitinen, H., Sinkkonen, J., Reinikainen, K., Alho, K., Lavikainen, J., Naatanen, R., 1993. Selective attention enhances the auditory 40$\mathrm{Hz}$ transient response in humans. Nature 364, 59-60.

Varela, F.J., 1995. Resonant cell assemblies: a new approach to cognitive function and neuronal synchrony. Biol. Res. 28, 81-95.

Vescovi, A.L., Parati, E.A., Gritti, A., Poulin, P., Ferrario, M., Wanke, E., Frölichsthal-Schoeller, P., Cova, L., Arcellana-Panlilio, M., Colombo, A., Galli, R., 1999. Isolation and cloning of multipotential stem cells from the embryonic human CNS and establishment of transplantable human neural stem cell lines by epigenetic stimulation. Exp. Neurol. 156, 71-83.

Weis, R., Müller, B., Fromherz, P., 1996. Neuron adhesion on silicon chip probed by an array of field-effect transistors. Phys. Rev. Lett. 76, 327-330.

Wilson, R.J., Breckenridge, L., Blackshaw, S.E., Connolly, P., Dow, J.A.T., Curtis, A.S.G., Wilkinson, C.D.W., 1994. Simultaneous multisite recordings and stimulation of single isolated leech neurons using planar extracellular electrode arrays. Neurosci. Methods 53, 101-110.

Zbilut, J.P., Thomasson, N., Webber Jr., C.L., 2002. Recurrence quantification analysis as a tool for nonlinear exploration of nonstationary cardiac signals. Med. Eng. Phys. 24, 53-60.

Zbilut, J.P., Webber, C.L., 1992. Embeddings and delays as derived from quantification of recurrent plots. Phys. Lett., 171.

Zeck, G., Fromherz, P., 2001. Noninvasive neuroelectronic interfacing with synaptically connected snail neurons on a semiconductor chip. Proc. Natl. Acad. Sci. 98, 10457-10462. 